Check for updates

Cite this: RSC Adv., 2021, 11, 7925

Received 30th September 2020 Accepted 3rd February 2021

DOI: $10.1039 / \mathrm{dOra0} 8372 \mathrm{k}$

rsc.li/rsc-advances

\section{Gamma radiation shielding characteristics of various spinel ferrite nanocrystals: a combined experimental and theoretical investigation}

\author{
Rajkumar M. Lokhande, ${ }^{* a}$ Vithal Vinayak, $^{b}$ Sachin V. Mukhamale \\ and Pankaj P. Khirade (iD *d
}

\begin{abstract}
This work presents the facile synthesis of $\mathrm{Ni}, \mathrm{Mn}, \mathrm{Zn}, \mathrm{Cu}$ and $\mathrm{Co}$ spinel ferrite nanocrystals via sol-gel auto-ignition and the investigation of their structural and gamma ray shielding characteristics. Experimentally, gamma ray shielding parameters are determined with different gamma ray sources and $\mathrm{Nal}(\mathrm{Tl})$ scintillation detector and theoretically via Monte-Carlo simulation (Geant4) as well as NIST-XCOM database. X-ray diffractograms elucidate the cubic spinel structure without any contaminating phases for all synthesized nano-ferrites. TEM results evidence the formation of ultrafine crystallites in nano-regime dimensions. Nanocrystalline spinel ferrites in pellet form have been exposed to gamma radiation from diverse sources by changing the radiation dose intensity. The comparative study of the linear attenuation coefficient, mass attenuation coefficient, total atomic cross section, total electronic cross section, effective atomic number, effective electron density and half value layer for manufactured spinel ferrites is carried out using NIST-XCOM and Geant4 at 122-1330 keV. Gamma ray energy absorption buildup factor (EABF) is investigated for five selected ferrites at $100 \mathrm{keV}$ to $1500 \mathrm{keV}$ incident photon energy and penetration depth from 1 to 40 mfp using geometric progression (G-P) fitting technique. EABF is found to be maximum at an intermediate region, mainly attributed to the Compton scattering process. Zinc ferrite exhibits a higher value of EABF among other ferrites, which mainly depends on the chemical composition of the material and crystallite size effect. The EABF is investigated as a function of penetration depth and is found to be maximum for a penetration depth of $40 \mathrm{mfp}$. Experimental and theoretical simulation results are found to be in good agreement. The Monte-Carlo simulation of radiation interaction with materials has evidenced to be an excellent approximation tool in exploring spinel ferrite performance in radiation atmosphere.
\end{abstract}

\section{Introduction}

Nanotechnology is one of the fastest growing scientific fields with applications in many diverse areas, including electronics. The term nanoelectronics refers to the use of nanotechnology in electronic components with critical dimensions and size ranging between $1 \mathrm{~nm}$ and $100 \mathrm{~nm} .^{1}$ All electronics devices manufactured by humans are continuously exposed to diverse types of radiation from natural sources as well as man-made sources. The miniaturization of electronic devices and

${ }^{a}$ Department of Physics, Shirish Madhukarrao Chaudhari College, Jalgaon, MS, 425001, India.E-mail: rajml@gmail.com

${ }^{b}$ Department of Chemistry, Shri Chhatrapati Shivaji College, Omerga, Osmanabad, MS, 413606, India

${ }^{c}$ Department of Physics, Shri Pundlik Maharaj Mahavidyalaya, Nandura Rly, MS, 443404, India

${ }^{d}$ Department of Physics, Shri Shivaji Science College, Amravati, MS, 444603, India. E-mail: pankajkhirade@gmail.com growing integration of electronic components may have an undesirable impact on the component sensitivity towards ionizing radiations. ${ }^{2}$

Currently, spinel ferrites of nano-dimensions are technologically trending materials due to their distinct electromagnetic characteristics. ${ }^{3-5}$ Spinel ferrites $\left(\mathrm{M}-\mathrm{Fe}_{2} \mathrm{O}_{4}\right)$ have several applications such as in transformers, inductors, capacitors, isolators, circulators, gyrators, phase shifters, reconfigurable antennas, spintronics memory devices, wireless mobile communication, and biomedical instrumentation. ${ }^{6-10}$

Gamma rays are a kind of electromagnetic radiation, and are the packets of energy called photons $(h v)$ emitted by the nucleus of some radionuclides resulting in radioactive decay. Gamma rays are most energetic photons in the electromagnetic spectrum having energies beyond $100 \mathrm{keV} .{ }^{11}$ Gamma radiation interacts with matter through ionization through three phenomena: photoelectric effect, Compton scattering and pair production. The enormously high energy of gamma rays permits them to enter just about everything. ${ }^{12}$ They can even 
pass-through skin, teeth, and bones and destroy living cells, produce gene mutations which cause cancer. This makes gamma rays extremely hazardous. ${ }^{13}$ Gamma radiation is powerful and can affect most electrical components. Simple equipment including motors, switches, incandescent lights, wiring, and solenoid are fairly radiation resistant and might never show any radiation impacts, even after exposure to large radiation. However, diodes and computer chips are much more sensitive to gamma radiation. Diodes and computer chips will show very slight functional damage up to about 50 to $100 \mathrm{~Sv}{ }^{\mathbf{1 4 , 1 5}}$

The effect of gamma radiation on electronic memory devices has been investigated by I. Fetahovic et al. ${ }^{16}$ The impact of direct ionizing radiation on semiconductor memory performance has been scrutinized by using Monte-Carlo simulation technique. The internal crystalline structure of the materials of electronic components is disrupted on interaction with gamma radiation. The functioning of electronic components breaks down and then fails when they are exposed to significant gamma radiation.

Ionizing radiation creates hole-electron couples in the electronic components, changes the transistor parameters and eventually destroys them. It can also cause leakage currents among circuits. As radiation particles voyage through a material they transfer part of their energies to the electrons and the nucleus of the material and rupture the chemical bonds, producing ionization and atomic displacement. ${ }^{17}$ For example, proton damage to a transistor consists of ionization and results in damage. ${ }^{18}$

In order to protect the electronic devices from gamma radiation several approaches are being established. Likewise, some electronics can be toughened so that they are not influenced as much by larger gamma radiation doses by providing shielding or by coating with radiation-resistant materials. ${ }^{19}$ Most nuclear reactors and electronic sectors can only maintain their functionality in radioactive environments with tungsten or lead shielding. ${ }^{20}$ It is heavy and bulky, and its design, installation and replacement are complex and expensive processes. The mass attenuation coefficient, total atomic cross section, total electronic cross section, energy absorption buildup factor (EABF) and exposer buildup factor (EBF) are the basic parameters for measuring the interaction of radiation with matter, which can be applied for the shielding purposes. $^{21}$

In the literature, the comparison of the experimental results on nanomaterials, soils, glass materials, composite materials, different detector materials, rocks and concrete, polymer, and biomaterials with the NIST-XCOM (photon cross sections database) and Monte-Carlo simulations is available. ${ }^{22,23}$

R. H. Kadam et al. have determined the mass attenuation coefficient of magnesium ferrite produced via ceramic method. ${ }^{24}$ The Hubbel's mixture rule is utilized to find the mass absorption coefficient and related parameters for the prepared $\mathrm{MgFe}_{2} \mathrm{O}_{4} \cdot{ }^{25} \mathrm{~S}$. D. Raut et al. have investigated the gamma ray energy absorption and exposure buildup aspects of $\mathrm{Co} / \mathrm{Zn} / \mathrm{Ni}$ / $\mathrm{Mg}$ spinel ferrites utilizing G-P fitting method in the energy range of $0.015-15.00 \mathrm{MeV}$ up to the penetration depth of 40 mfp. ${ }^{26}$
However, there are no noteworthy literature available on the shielding characteristics of spinel ferrite electronic materials. Monte-Carlo simulation is found to be the most effective tool to determine the radiation interaction parameters in diverse kinds of materials, compounds and composites for shielding properties. Demonstrating the photon attenuation properties of materials via computer software offers superior accuracy and flexibility of use than the experimental procedure. There are many Monte-Carlo simulation codes available for the study of radiation transport, particle physics, medical physics, cosmo-physics, radiotherapy, and radiation biology, namely, MCNP, GEANT4 and FLUKA. ${ }^{27-29}$ Geant4 is a MonteCarlo simulation code based on $\mathrm{C}++$, an object-oriented programming method, and is freely available. Geant 4 code is applicable for measurement of approximation of photon mass attenuation coefficients for different types of most common scintillation crystal detectors at different energies. ${ }^{30}$

The aim of this research work is to fabricate single-phase nanocrystalline spinel ferrite materials via the ecofriendly sol-gel technique and to inspect the performance of these materials when exposed to gamma radiation in order to establish their functionality in a radiation atmosphere. The experimental procedure has been used to test the radiation attenuation parameters of spinel ferrites. Diverse kinds of spinel ferrites viz. nickel ferrite $\left(\mathrm{NiFe}_{2} \mathrm{O}_{4}\right)$, manganese ferrite $\left(\mathrm{MnFe}_{2} \mathrm{O}_{4}\right)$, zinc ferrite $\left(\mathrm{ZnFe}_{2} \mathrm{O}_{4}\right)$, copper ferrite $\left(\mathrm{CuFe}_{2} \mathrm{O}_{4}\right)$ and cobalt ferrite $\left(\mathrm{CoFe}_{2} \mathrm{O}_{4}\right)$ have been exposed to ionizing gamma radiation by varying the radiation dose intensity. The impact of direct ionizing radiation on spinel ferrite materials' shielding characteristics has been explored by using MonteCarlo simulation (Geant4) and NIST-XCOM database. Also, a theoretical study has been undertaken to get information on the energy absorption buildup factor of five ferrite materials using G-P fitting method at incident photon energy of $100 \mathrm{keV}$ to $1500 \mathrm{keV}$ up to penetration depths of $40 \mathrm{mfp}$. The obtained radiological data can be very much useful for elucidating the gamma ray shielding characteristics of spinel ferrite electronic materials.

\section{Experimental and calculation method}

\subsection{Synthesis of nanocrystalline spinel ferrite}

Materials. Analytical reagent (AR) grade nickel nitrate $\left(\mathrm{Ni}\left(\mathrm{NO}_{3}\right)_{2} \cdot 6 \mathrm{H}_{2} \mathrm{O}\right)$, manganese nitrate $\left(\mathrm{Mn}\left(\mathrm{NO}_{3}\right)_{2} \cdot 6 \mathrm{H}_{2} \mathrm{O}\right)$, zinc nitrate $\left(\mathrm{Zn}\left(\mathrm{NO}_{3}\right)_{2} \cdot 6 \mathrm{H}_{2} \mathrm{O}\right)$, copper nitrate $\left(\mathrm{Cu}\left(\mathrm{NO}_{3}\right)_{2} \cdot 6 \mathrm{H}_{2} \mathrm{O}\right)$, cobalt nitrate $\left(\mathrm{Co}\left(\mathrm{NO}_{3}\right)_{2} \cdot 6 \mathrm{H}_{2} \mathrm{O}\right)$, ferric nitrate $\left(\mathrm{Fe}\left(\mathrm{NO}_{3}\right)_{3} \cdot 9 \mathrm{H}_{2} \mathrm{O}\right)$ and L-ascorbic acid $\left(\mathrm{C}_{6} \mathrm{H}_{8} \mathrm{O}_{6}\right)$ used as precursors with $99.9 \%$ purity were procured from Sigma-Aldrich and used as received without further distillation.

\subsection{Synthesis and characterizations}

Nanocrystalline spinel ferrite materials viz. nickel ferrite $\left(\mathrm{NiFe}_{2} \mathrm{O}_{4}\right)$, manganese ferrite $\left(\mathrm{MnFe}_{2} \mathrm{O}_{4}\right)$, zinc ferrite $\left(\mathrm{ZnFe}_{2} \mathrm{O}_{4}\right)$, copper ferrite $\left(\mathrm{CuFe}_{2} \mathrm{O}_{4}\right)$ and cobalt ferrite $\left(\mathrm{CoFe}_{2} \mathrm{O}_{4}\right)$, were efficiently produced via the sol-gel auto-ignition technique and 
L-ascorbic acid as a combustion agent. The AR grade nitrates of $\mathrm{Ni}, \mathrm{Mn}, \mathrm{Zn}, \mathrm{Cu}$, Co and L-ascorbic acid were liquified in distilled water distinctly to accomplish a homogenous solution. The precursor metal nitrates were dissolved together with the least amount of distilled water essential to achieve a clear solution. The reaction procedure was done in an air atmosphere deprived of the shield of inert gases. The metal nitrates to combustion agent (L-ascorbic acid) ratio was preserved as $1: 3$. Liquified ammonia was added dropwise to maintain the $\mathrm{pH}$ at the neutral value 7 . The auto-ignition reaction was carried out for few hours with continuous thermal treatment at $100{ }^{\circ} \mathrm{C}$ to produce the required product. The as-prepared powders were sintered at $750{ }^{\circ} \mathrm{C}$ for $6 \mathrm{~h}$ and utilized for further examinations. The sintered powder samples were assorted with polyvinyl alcohol (PVA) mediator as a binder and hard-pressed into compact cylindrical pellets with dimensions of $10 \mathrm{~mm}$ diameter and less than $3 \mathrm{~mm}$ thickness by the isostatic pressing method under a pressure of $550 \mathrm{~kg} \mathrm{~cm} \mathrm{~cm}^{-2}$. The pellets were heat-treated in a furnace at $430{ }^{\circ} \mathrm{C}$ for $2 \mathrm{~h}$ for the exclusion of PVA and then used for radiation exposure.

Xray diffraction (XRD) analysis was conducted on a PANalytical X'pert pro-diffractometer. The wavelength of the X-ray was $1.542 \AA$ (Cu- $\mathrm{K} \alpha$ radiation, $40 \mathrm{kV}$ and $100 \mathrm{~mA}$ ), in the $2 \theta$ scale of $20^{\circ}-80^{\circ}$ and at the scanning rate of $0.02^{\circ}$. The brightfield surface topography of the samples and particle size distribution were obtained by transmission electron microscopy (TEM, CM-200 FEG PHILIPS).

\subsection{Experimental procedures}

The synthesized nanocrystalline spinel ferrites in pellet form were irradiated with gamma rays generated by the radioactive sources, ${ }^{57} \mathrm{Co},{ }^{133} \mathrm{Ba},{ }^{22} \mathrm{Na},{ }^{137} \mathrm{Cs},{ }^{54} \mathrm{Mn}$ and ${ }^{60} \mathrm{Co}$, via the narrow beam geometry setup $\mathrm{NaI}(\mathrm{Tl})$ scintillation detector. All these radioactive sources acquired from Bhabha Atomic Research Centre (BARC), Mumbai, India producing different energies viz. ${ }^{57} \mathrm{Co}(122 \mathrm{keV}),{ }^{133} \mathrm{Ba}(356 \mathrm{keV}),{ }^{22} \mathrm{Na}$ (511 and $\left.1275 \mathrm{keV}\right),{ }^{137} \mathrm{Cs}$ (662 keV), ${ }^{54} \mathrm{Mn}(835 \mathrm{keV})$ and ${ }^{60} \mathrm{Co}(1173$ and $1332 \mathrm{keV})$ were collimated. The angle between a radioactive source and the pellet materials was kept as $90^{\circ}$ for narrow beam analysis. The NaI scintillation detector with narrow beam geometry was utilized as the gamma ray spectroscopic system. The analysis of the amplified signals by selecting a spectrometry system included a $\left(2^{\prime \prime} \times 2^{\prime \prime}\right) \mathrm{NaI}(\mathrm{Tl})$ crystal with an energy resolution of $8.2 \%$ at $662 \mathrm{keV}$ from the gamma decay of ${ }^{137} \mathrm{Cs}$. The peak measurement energy versus count depends on full width at half maxima (FWHM) and the $8 \mathrm{~K}$ multichannel analyzer. The diameters of the pellets played an important role in the narrow beam system (source-sample-detector) and were determined using a traveling microscope for more accuracy. The samples were put in a lead-shielded narrow beam geometry set up (source-sample-detector). The sample thickness was selected in order to satisfy the following ideal condition. ${ }^{31}$

$$
2 \leq \ln \left(\frac{I_{0}}{I}\right) \leq 4
$$

There are two steps in determining the attenuation coefficient by experiment; first to calculate with the source and without the sample and then with the source and with the sample. The present experiments were carried out in an airconditioned room to avoid possible shifts of the photo-peaks. The room temperature was maintained at $20 \pm 1{ }^{\circ} \mathrm{C}$ throughout the experiment.

Determination of mass attenuation coefficient. According to Beer-Lambert law a parallel ray of X-ray or Gamma ray photons passing through matter is attenuated due to an electromagnetic region (absorption and scattering) and mathematically expressed as: ${ }^{32}$

$$
I=I_{0} \exp \left(-\mu_{\mathrm{m}} t\right)
$$

where $I_{0}$ and $I$ are the incident and transmitted photon intensities of gamma radiations, $\mu_{\mathrm{m}}$ is the mass attenuation coefficient of the nanocrystalline spinel ferrites and $t$ is the thickness of the sample. Then mixture rule is applied for a compound or mixture of elements.

$$
\mu_{\mathrm{m}}=\sum i w_{i}\left(\mu_{\mathrm{m}}\right)_{i}
$$

where $w_{i}$ and $\left(\mu_{\mathrm{m}}\right)_{i}$ are the weight fraction and mass attenuation coefficient of the $i^{\text {th }}$ constituent of the ferrites, respectively. The weight fraction $\left(w_{i}\right)$ of different chemical compositions is given by

$$
w_{i}=\frac{n_{i} A_{i}}{\sum j n_{j} A_{j}}
$$

Determination of total atomic and electronic cross section. The total atomic cross section $\left(\sigma_{\mathrm{t}, \mathrm{a}}\right)$ can be written mathematically as, ${ }^{33}$

$$
\sigma_{\mathrm{t}, \mathrm{a}}=\frac{\left(\mu_{\mathrm{m}}\right)_{\text {ferrite }}}{N_{\mathrm{A}} \sum_{i}^{n}\left(w_{i} / A_{i}\right)}
$$

where $N_{\mathrm{A}}$ is the Avogadro's number and $A_{i}$ is the atomic weight of the $i^{\text {th }}$ constituent element of the spinel ferrite. Likewise, the total electronic cross section $\left(\sigma_{\mathrm{t}, \mathrm{el}}\right)$ is mathematically expressed as,

$$
\sigma_{\mathrm{t}, \mathrm{el}}=\frac{1}{N_{\mathrm{A}}} \sum_{i} \frac{f_{i} A_{i}}{Z_{i}}\left(\mu_{\mathrm{m}}\right)_{i}
$$

where $f_{i}$ is the number of atoms of the $i^{\text {th }}$ constituent element relative to the total number of atoms of the spinel ferrites and $Z_{i}$ is the atomic number of the $i^{\text {th }}$ element in the material.

Effective atomic number $\left(Z_{\text {eff }}\right)$. The effective atomic number can be estimated using the equation

$$
Z_{\text {eff }}=\frac{\sigma_{\mathrm{t}, \mathrm{at}}}{\sigma_{\mathrm{t}, \mathrm{el}}}
$$

Effective electron density $\left(N_{\text {eff }}\right)$.

$$
N_{\text {eff }}=\left(\frac{N_{\mathrm{A}}}{A_{\text {eff }}}\right) \times Z_{\text {eff }}
$$




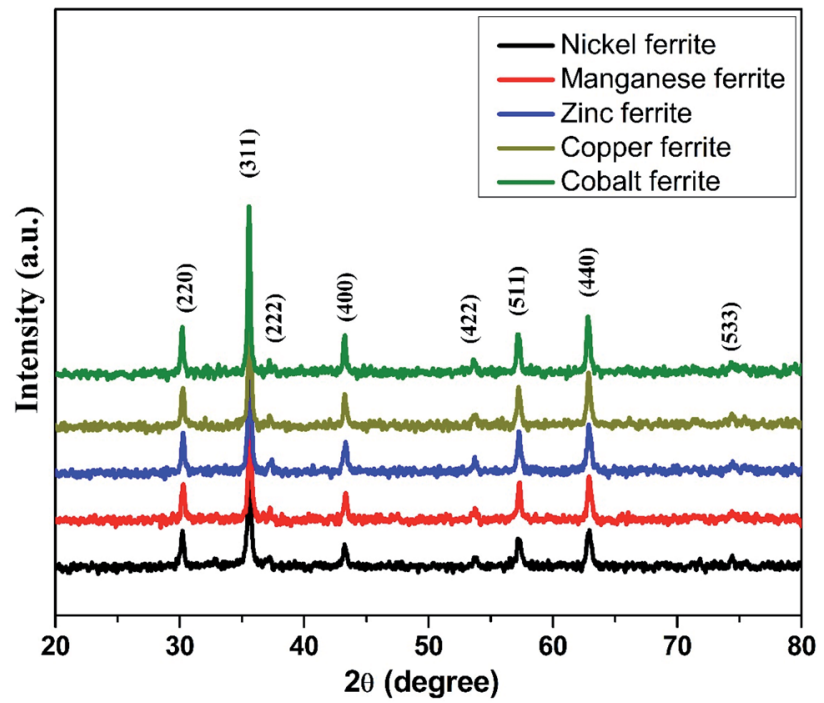

Fig. 1 X-ray diffractograms for the produced spinel nano-ferrites.

where $A_{\text {eff }}$ is the effective atomic mass also recognized as the ratio of atomic weight and total number of atoms.

Half value layer (HVL). The half value layer is mathematically expressed as ${ }^{34}$

$$
\mathrm{HVL}=\frac{\ln 2}{\mu}=\frac{0.693}{\mu}
$$

Here, $\mu$ is the linear attenuation coefficient. The $\mu$ measured experimentally from the narrow beam geometry setup of $\mathrm{NaI}(\mathrm{Tl})$ detector results from the energy and counts observed on the monitor of the system.

Determination of energy absorption buildup factor (EABF). Several researchers studied the energy absorption and exposure buildup factor (EABF) with the help of the G-P fitting technique. ${ }^{35}$ Harima et al. developed the G-P fitting technique with the aid of NIST datasheets and ANSI-6.4.3 and illustrated the energy absorption and exposure buildup factor up to the penetration depth of $40 \mathrm{mfp}$ at 0.015 to $15 \mathrm{MeV}$ incident photon energy. ${ }^{36}$ There are three key stages in the determination of the EABF: (a) the determination of equivalence atomic number $\left(Z_{\text {eq }}\right)$, (b) the determination of G-P fitting parameters and (c) the computation of EABF. The value of $Z_{\text {eq }}$ depends on the NISTXCOM database, partial attenuation coefficient $\left(\mu_{\mathrm{m}}\right)_{\text {comp }}$ and total attenuation coefficient $\left(\mu_{\mathrm{m}}\right)_{\mathrm{total}}$. Statistically, $Z_{\mathrm{eq}}$ is written in the following form: ${ }^{37}$

$$
Z_{\mathrm{eq}}=\frac{Z_{1}\left(\log R_{2}-\log R\right)+Z_{2}\left(\log R-\log R_{1}\right)}{\log R_{2}-\log R_{1}}
$$

The G-P fitting method is provided by the American National Standards (ANSI/ANS-6.4.3-1991). ${ }^{38}$ Using the interpolation formula, five G-P fitting parameters $\left(a, b, c, d\right.$ and $\left.X_{\mathrm{k}}\right)$ for the selected samples were computed at different incident photon energies (100-1500 keV) using equivalent atomic number $\left(Z_{\mathrm{eq}}\right)$ up to the penetration depth of $40 \mathrm{mfp}$. The interpolated values were obtained by using the following equation: ${ }^{39}$

$$
C=\frac{C_{1}\left(\log Z_{2}-\log Z_{\mathrm{eq}}\right)+C_{2}\left(\log Z_{\mathrm{eq}}-\log Z_{1}\right)}{\log Z_{2}-\log Z_{1}}
$$

The calculation of the energy absorption buildup factor with the assistance of the G-P fitting formula depends on the shielding thickness as given by following equations:

$$
B(E, X)=1+\frac{b-1}{K-1}\left(K_{\mathrm{x}}-1\right) \quad \text { at } K \neq 1
$$

$$
B(E, X)=1+(b-1) \quad \text { at } K=1
$$

$$
K(E, x)=c x^{a}+d \frac{\tan h\left(\frac{x}{X_{\mathrm{k}}}-2\right)-\tan h(-2)}{1-\tan h(-2)}
$$

where $b$ is the buildup factor at $1 \mathrm{mfp}$ and $K$ is a multiplication factor for dose through $1 \mathrm{mfp}$ photon penetration.

Monte-Carlo simulation. Geant4 is a platform for the simulation of the passage of particles through matter utilizing Monte Carlo methods. Geant4 is an object-oriented toolkit contingent on the $\mathrm{C}++$ programming language, which can be used for the measurement of radiation interaction with matter at a wide energy range of $250 \mathrm{eV}$ to $100 \mathrm{TeV}$. The study of electromagnetic radiation in the Geant4 environment is available for low electromagnetic (EM) test package; we studied the EM test 13, 15 and 18 for low EM interaction. The Geant 4 method for electromagnetic package was especially applied for narrow beam geometry. The study was carried out in the UNIX operating system Geant4 version 9.06.p01 for all gamma sources. The attenuation coefficient was calculated using computer environment and Geant4 application G4RunManager, the observed result was due to photo-electric effect, Compton scattering and

Table 1 Various structural parameters of nanocrystalline spinel ferrites

\begin{tabular}{lllll}
\hline Sample & Space group & $\begin{array}{l}\text { Average crystallite } \\
\text { size (nm) from XRD }\end{array}$ & $\begin{array}{l}\text { Crystallite size } \\
\text { (nm) from TEM }\end{array}$ & $\begin{array}{l}\text { Lattice parameter } \\
(\AA)\end{array}$ \\
\hline $\mathrm{NiFe}_{2} \mathrm{O}_{4}$ & $F d \overline{3} m$ & 36.25 & 34.02 & 8.3619 \\
$\mathrm{MnFe}_{2} \mathrm{O}_{4}$ & $F d \overline{3} m$ & 33.87 & 35.46 & 8.4742 \\
$\mathrm{ZnFe}_{2} \mathrm{O}_{4}$ & $F d \overline{3} m$ & 32.31 & 37.62 & 8.4516 \\
$\mathrm{CuFe}_{2} \mathrm{O}_{4}$ & $F d \overline{3} m$ & 39.75 & 42.54 & 8.3839 \\
$\mathrm{CoFe}_{2} \mathrm{O}_{4}$ & $F d \overline{3} m$ & 52.45 & 56.02 & 8.3891
\end{tabular}


pair production phenomenon. The primary information is required for the construction of the detector geometry of the electromagnetic package for Geant 4 simulation. There are three stages; in the first stage of construction, the narrow beam geometry set up of a monochromatic source exposes the selected gamma radiation to the material and detector and a unique distance between source-sample-detector is set. The second stage is the setting of the energy, here we have selected the energy regions of $122 \mathrm{keV}, 356 \mathrm{keV}, 511 \mathrm{keV}, 662 \mathrm{keV}, 1170$ $\mathrm{keV}, 1275 \mathrm{keV}$ and $1330 \mathrm{keV}$, chemical composition, density, elemental weight fraction and thicknesses of the selected spinel ferrites, and setting the physical processes of photoelectric effect, Compton (coherent and incoherent) scattering and pair production corresponding to photon energy. The third stage is

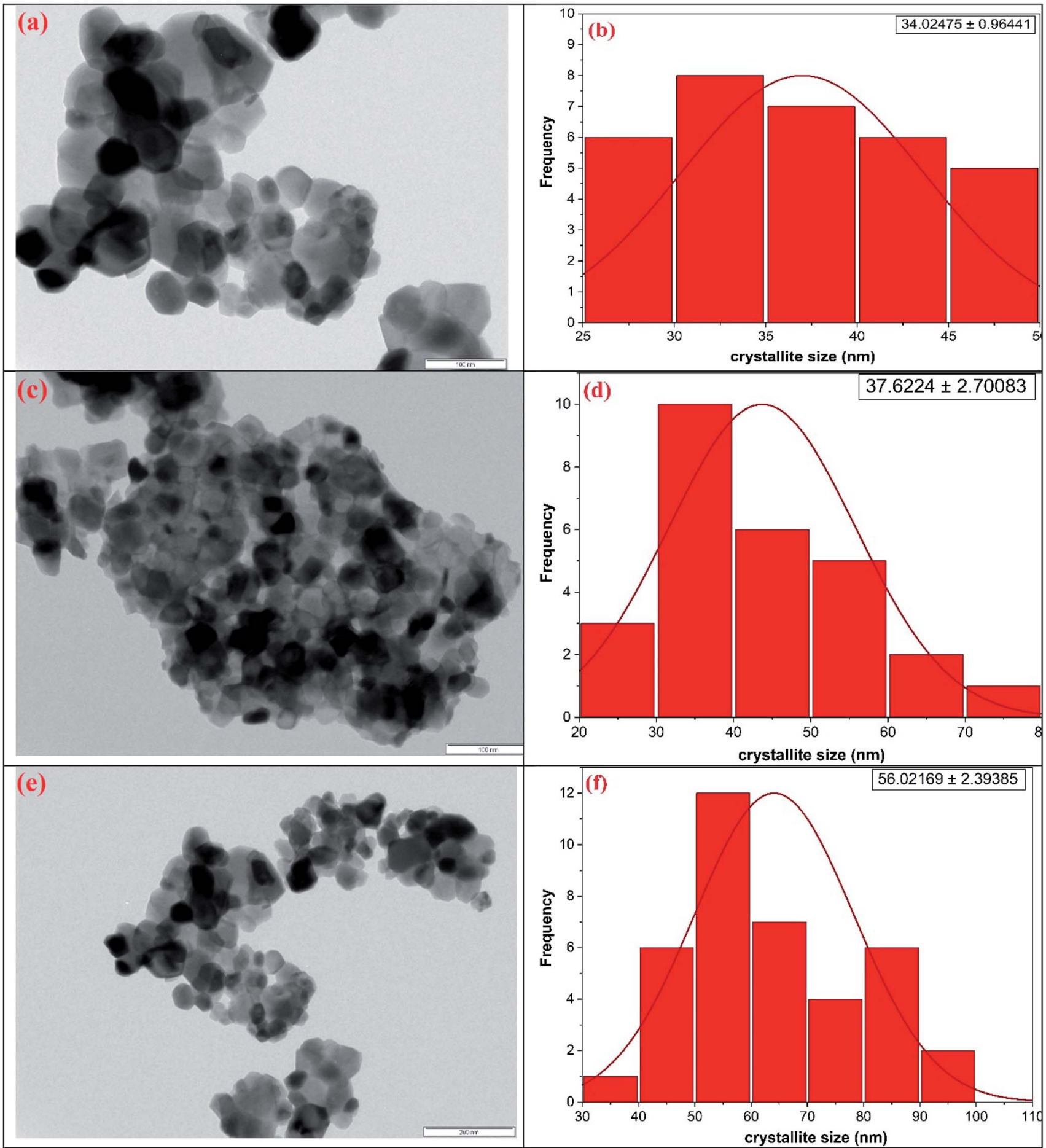

Fig. 2 TEM micrographs along with particle size distribution for the representative ( $a$ and b) nickel ferrite (c and d) zinc ferrite and (e and f) cobalt ferrite nanocrystals. 


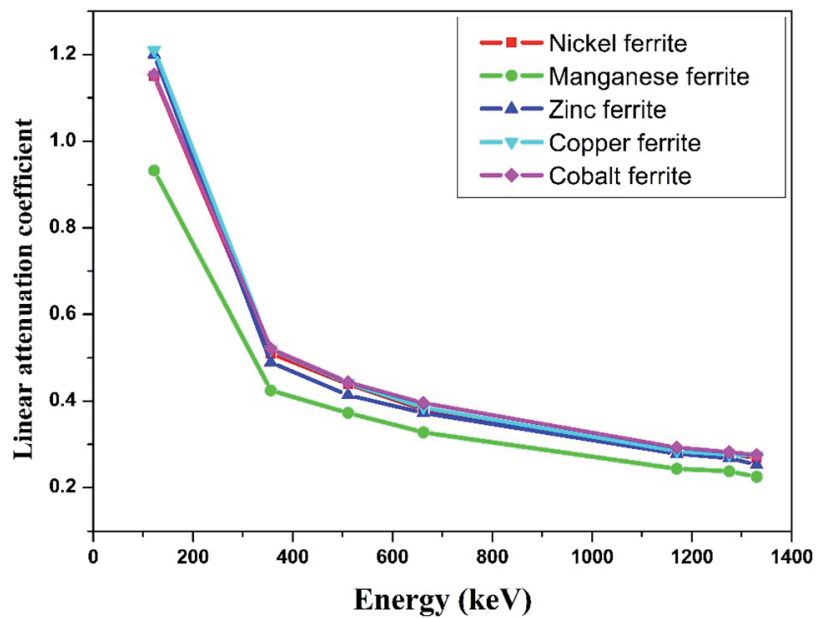

Fig. 3 Experimental linear attenuation coefficients $(\mu)$ versus incident photon energy.

the measurement of the simulation value by using GM calculator after $10^{6}$ hits of gamma radiation on the selected biomaterial at a particular thickness.

\section{Results and discussion}

The room-temperature X-ray diffraction (XRD) outlines of spinel ferrites synthesized via the sol-gel auto-ignition technique are presented in Fig. 1. The occurrence of a single intense peak at $2 \theta$ $\sim 35^{\circ}$ is usually related to the most intense (311) diffraction plane characteristics of cubic symmetry. All the reflections observed could be attributed to a cubic spinel lattice, without evidence of additional impurities, showing their single-phase structure. From the XRD profile, the average crystallite sizes of the samples were calculated by the Debye-Scherrer formula based on the diffraction peak of the highly intense (311) plane. ${ }^{40}$ The average crystallite size was found to be in the range of 32$52 \mathrm{~nm}$ for all the ferrite samples. The lattice parameter $(a)$ obtained by using XRD data was found to lie in the range of 8.3619-8.4742 $\AA$ for different spinel ferrites. The structural parameters are shown in Table 1.

The microstructural characteristics of the ferrite nanocrystals were exposed by employing transmission electron microscopy (TEM) as shown in Fig. 2(a-f). Fig. 2(a), (c) and (e) depicts the bright field TEM pictures of the representative nickel ferrite, zinc ferrite and cobalt ferrite nanocrystalline samples, respectively, while the adjacent plots, Fig. 2(b), (d) and (f), demonstrate the crystallite size distribution obtained using the statistical method (Gaussian fitting) from the TEM pictures. Also, the derived mean crystallite sizes are listed in Table 1. The TEM micrographs depict that the surface is composed of homogeneously distributed single and partially polycrystalline facetted grains of varying sizes in nano dimension scale.

We successfully carried out the narrow beam geometry experimental investigations on the manufactured nano-ferrites in pellet form using different gamma ray sources such as ${ }^{57} \mathrm{Co}$ (122 keV), ${ }^{133} \mathrm{Ba}(356 \mathrm{keV}),{ }^{22} \mathrm{Na}$ (511 and $\left.1275 \mathrm{keV}\right),{ }^{137} \mathrm{Cs}(662$ $\mathrm{keV}),{ }^{54} \mathrm{Mn}(835 \mathrm{keV})$ and ${ }^{60} \mathrm{Co}$ (1173 and $\left.1332 \mathrm{keV}\right)$ and $\mathrm{NaI}(\mathrm{Tl})$ detector. The linear attenuation coefficient $(\mu)$ for the synthesized nickel, manganese, zinc, copper and cobalt nano-ferrites was measured at room temperature for the evaluation of the shielding characteristics with respect to incident photon energy in the range of $122 \mathrm{keV}$ to $1330 \mathrm{keV}$.

The linear attenuation coefficient $(\mu)$ with respect to photon energy of all the selected nano-ferrites is graphically represented in Fig. 3. It can be observed that as the photon energy increases the linear attenuation coefficient decreases exponentially for all the synthesized nano-ferrites. Zinc and cobalt nanoferrites exhibit high values of $\mu$; however, manganese nanoferrite displays the lowest value. Also, zinc ferrite with more discrepancy rapidly decreases in the energy range of 122 to 356 $\mathrm{keV}$ and then linearly declines with energy compared to the other spinel ferrites. The linear attenuation coefficient is an imperative parameter for the measurement of the shielding characteristics of materials. ${ }^{41}$

The mass attenuation coefficient $\left(\mu_{\mathrm{m}}\right)$ was determined using eqn (1). The theoretical mass attenuation coefficient was simulated by the Monte-Carlo simulation (Geant4) method as well as X-Com (NIST) database as shown in Table 2. The experimental, theoretical and simulated values of the mass attenuation coefficient fairly match with each other for all the synthesized nano-ferrites. Fig. 4 depicts the mass attenuation coefficient $\left(\mu_{\mathrm{m}}\right)$ versus incident photon energy (keV) plot of the representative zinc ferrite sample determined using experimental and theoretical (viz. Monto-Carlo simulation and XCOM) techniques, which exhibits the analogous nature. The

Table 2 Mass attenuation coefficient $\left(\mathrm{cm}^{2} \mathrm{~g}^{-1}\right)$ for the synthesized spinel nano-ferrites

\begin{tabular}{|c|c|c|c|c|c|c|c|c|c|c|c|c|c|c|c|}
\hline Energy (keV) & Geant4 & NIST & EXPT & Geant4 & NIST & EXPT & Geant4 & NIST & EXPT & Geant4 & NIST & EXPT & Geant4 & NIST & EXPT \\
\hline 356 & 0.0980 & 0.1010 & 0.0951 & 0.0966 & 0.0993 & 0.0921 & 0.0976 & 0.1006 & 0.0942 & 0.0973 & 0.1001 & 0.0961 & 0.0970 & 0.0998 & 0.0943 \\
\hline 511 & 0.0839 & 0.0848 & 0.0818 & 0.0828 & 0.0836 & 0.0809 & 0.0832 & 0.0842 & 0.0796 & 0.0830 & 0.0840 & 0.0818 & 0.0830 & 0.0839 & 0.0802 \\
\hline 662 & 0.0747 & 0.0751 & 0.0708 & 0.0739 & 0.0741 & 0.0711 & 0.0741 & 0.0744 & 0.0717 & 0.0740 & 0.0742 & 0.0712 & 0.0740 & 0.0743 & 0.0715 \\
\hline 1330 & 0.0532 & 0.0531 & 0.0499 & 0.0526 & 0.0525 & 0.0488 & 0.0527 & 0.0526 & 0.0486 & 0.0526 & 0.0525 & 0.0507 & 0.0527 & 0.0526 & 0.0498 \\
\hline
\end{tabular}




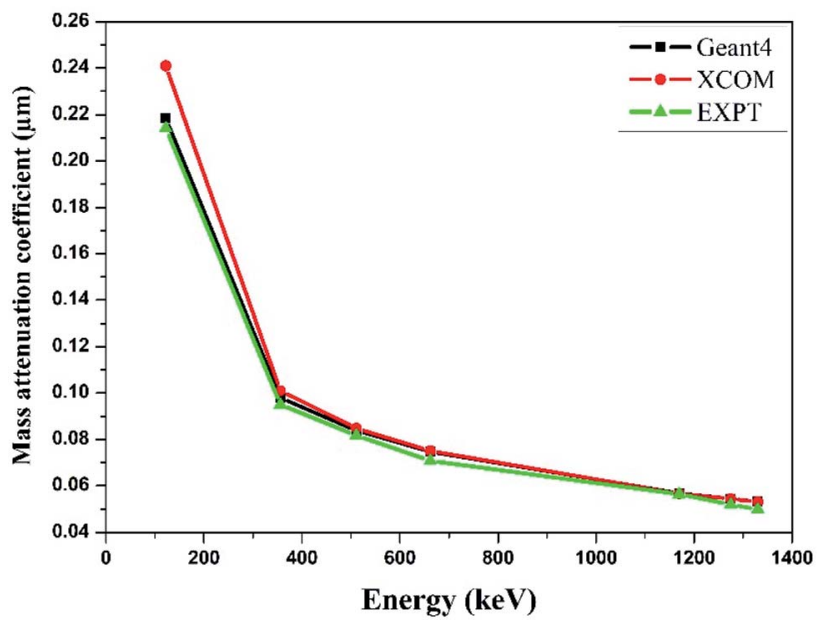

Fig. 4 Comparison of experimental and theoretical mass attenuation coefficient against incident photon energy for zinc ferrite.

same trend was observed for all the fabricated nano-ferrites. The value of the mass attenuation coefficient primarily depends on the incident photon energy, and it suddenly decreases with energy $(E<400 \mathrm{keV})$ as photoelectric effect is dominant here; at the moderate energy region the curve remains continual due to Compton scattering phenomenon as reported by Medhat et al. ${ }^{42}$ It reflects that Monte-Carlo simulation, experimental and theoretical outcomes are in good agreement with each other. The Monte-Carlo simulation results were more effective than the NIST-XCOM data.
Nano-magnesium ferrite has greater linear and mass attenuation coefficients compared to that of bulk magnesium ferrite synthesized via the standard ceramic technique reported in the literature. ${ }^{24}$ This could be accredited to the homogenous distribution of nano-crystallites with high electron density, which results in higher interaction probability between incident photons and nanocrystals compared to that with bulk ferrite.

Likewise, A. M. El-Khatib et al. investigated the gamma attenuation coefficients of micro-sized and nano-sized cadmium oxide (CdO) particles mixed with high-density polyethylene (HDPE) prepared by the compression molding technique. $^{43}$ They observed superior gamma ray shielding characteristics in nano-dimension cadmium oxide compared with that in micro-dimension cadmium oxide.

The effect of particle size on the gamma radiation shielding property of gadolinium oxide dispersed epoxy resin matrix composite was studied by Ran Li et al. ${ }^{44}$ They concluded that the produced nano- $\mathrm{Gd}_{2} \mathrm{O}_{3}$ reinforced epoxy composite shows both good shielding and mechanical properties as compared to micro-fillers and suggested this material as a promising novel shielding material for radiation protection purpose.

Similarly, we obtained the total atomic cross section $\left(\sigma_{t, \text { at }}\right)$ and total electronic cross section $\left(\sigma_{\mathrm{t}, \mathrm{el}}\right)$ parameters of the nanoferrites with the help of eqn (4) and (5), respectively. The experimental and theoretical values of $\sigma_{\mathrm{t}, \mathrm{at}}$ and $\sigma_{\mathrm{t} \text {,el }}$ for all the synthesized nano-ferrites are tabulated in Tables 3 and 4 , respectively. The graphical representation of the total atomic

Table 3 Total attenuation cross section $\sigma_{\mathrm{t}, \text { at }}$ (barn per atom) for the synthesized spinel nano-ferrites

\begin{tabular}{|c|c|c|c|c|c|c|c|c|c|c|c|c|c|c|c|}
\hline \multirow[b]{2}{*}{ Energy (keV) } & \multicolumn{3}{|c|}{ Nickel ferrite } & \multicolumn{3}{|c|}{ Manganese ferrite } & \multicolumn{3}{|c|}{ Zinc ferrite } & \multicolumn{3}{|c|}{ Copper ferrite } & \multicolumn{3}{|c|}{ Cobalt ferrite } \\
\hline & Geant4 & NIST & EXPT & Geant4 & NIST & EXPT & Geant4 & NIST & EXPT & Geant4 & NIST & EXPT & Geant4 & NIST & EXPT \\
\hline
\end{tabular}

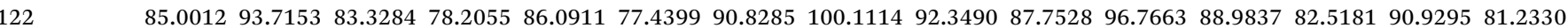

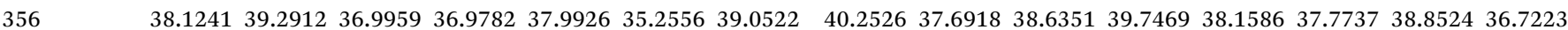

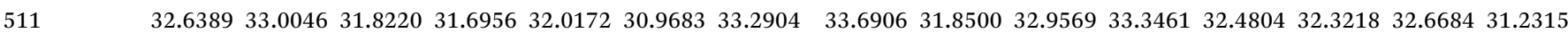

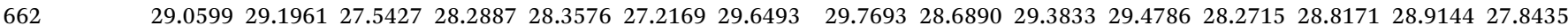

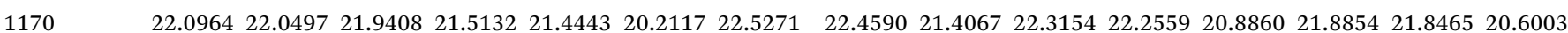

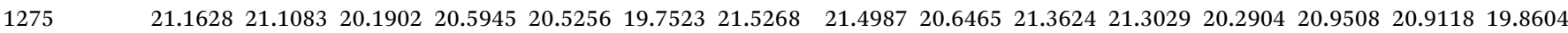

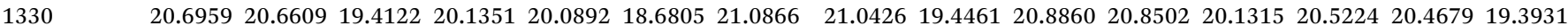

Table 4 Total electronic cross section $\sigma_{\mathrm{t}, \text { el }}$ (barn per atom) for the synthesized spinel nano-ferrites

\begin{tabular}{|c|c|c|c|c|c|c|c|c|c|c|c|c|c|c|c|}
\hline Energy (keV) & Geant4 & NIST & EXPT & Geant4 & NIST & EXPT & Geant4 & NIST & EXPT & Geant4 & NIST & EXPT & Geant4 & NIST & EXPT \\
\hline 356 & 2.3621 & 2.4344 & 2.2922 & 2.2714 & 2.3337 & 2.1656 & 2.0554 & 2.1186 & 1.9838 & 2.4375 & 2.5077 & 2.4075 & 2.2220 & 2.2854 & 2.1601 \\
\hline 511 & 2.0222 & 2.0449 & 1.9716 & 1.9469 & 1.9667 & 1.9022 & 1.7521 & 1.7732 & 1.6763 & 2.0793 & 2.1039 & 2.0492 & 1.9013 & 1.9217 & 1.8371 \\
\hline 662 & 1.8005 & 1.8089 & 1.7065 & 1.7376 & 1.7419 & 1.6718 & 1.5605 & 1.5668 & 1.5099 & 1.8538 & 1.8598 & 1.7837 & 1.6951 & 1.7008 & 1.6379 \\
\hline 1330 & 1.2823 & 1.2801 & 1.2027 & 1.2368 & 1.2340 & 1.1475 & 1.1098 & 1.1075 & 1.0235 & 1.3177 & 1.3155 & 1.2701 & 1.2072 & 1.2040 & 1.1408 \\
\hline
\end{tabular}




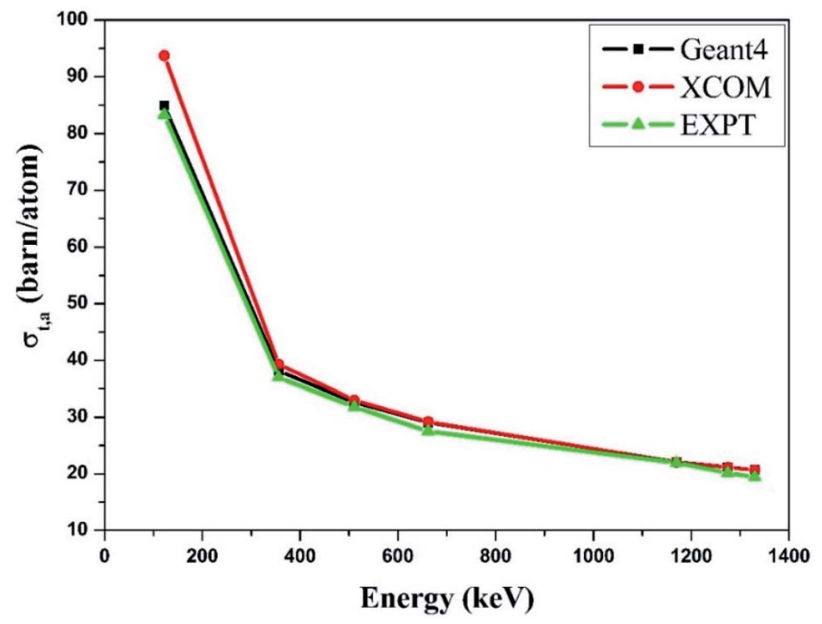

Fig. 5 Comparative study of the total atomic cross-section $\left(\sigma_{t, a}\right)$ verses incident photon energy for zinc ferrite.

cross section $\left(\sigma_{\mathrm{t}, \mathrm{at}}\right)$ and total electronic cross section $\left(\sigma_{\mathrm{t}, \mathrm{el}}\right)$ of the representative zinc ferrite sample are revealed in Fig. 5 and Fig. 6, respectively. Fig. 5 and 6 show that the experimental results are in good agreement with the Geant4 and XCOM outcomes. Also, they reflect that both $\sigma_{\mathrm{t}, \mathrm{at}}$ and $\sigma_{\mathrm{t}, \mathrm{el}}$ decrease with increasing incident photon energy.

The effective atomic number $\left(Z_{\text {eff }}\right)$ was estimated by using eqn (6). The value of $Z_{\text {eff }}$ is graphically represented in Fig. 7 for all the synthesized nano-ferrites against photon energy. It can be observed in Fig. 7 that the value of $Z_{\text {eff }}$ is declining with rise in photon energy (122-1330 keV). The decreasing behavior of $Z_{\text {eff }}$ with increasing energy is mainly because the photons live longer due to the photoelectric effect, Compton effect and pair production phenomenon. ${ }^{45}$ The nano-ferrites absorb more photons at higher energy and absorb minimum at lower energy. It reflects that at higher energy the Compton process is dominant, therefore, $Z_{\text {eff }}$ has a lower value due to the recoiling

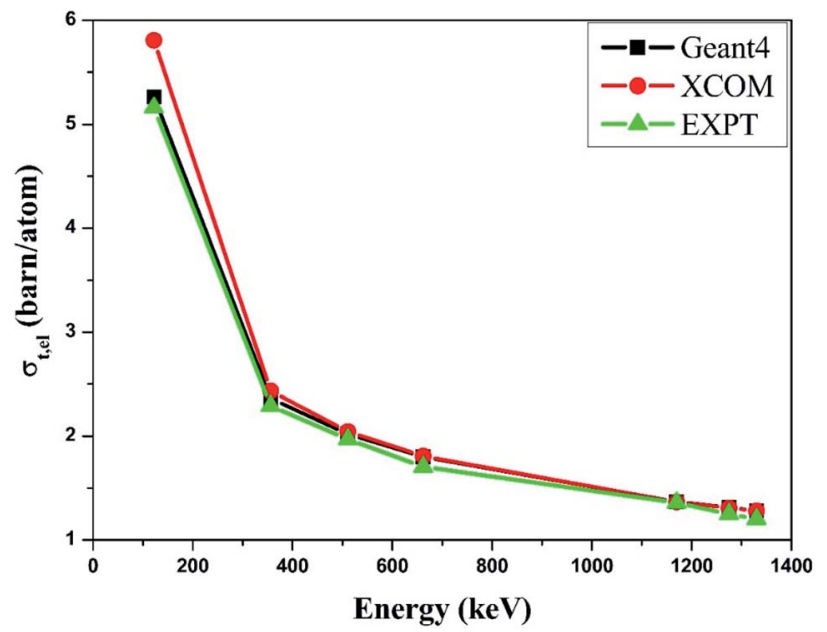

Fig. 6 Comparative study of the total electronic cross-section $\left(\sigma_{\mathrm{t}, \mathrm{e}}\right)$ versus incident photon energy of zinc ferrite.

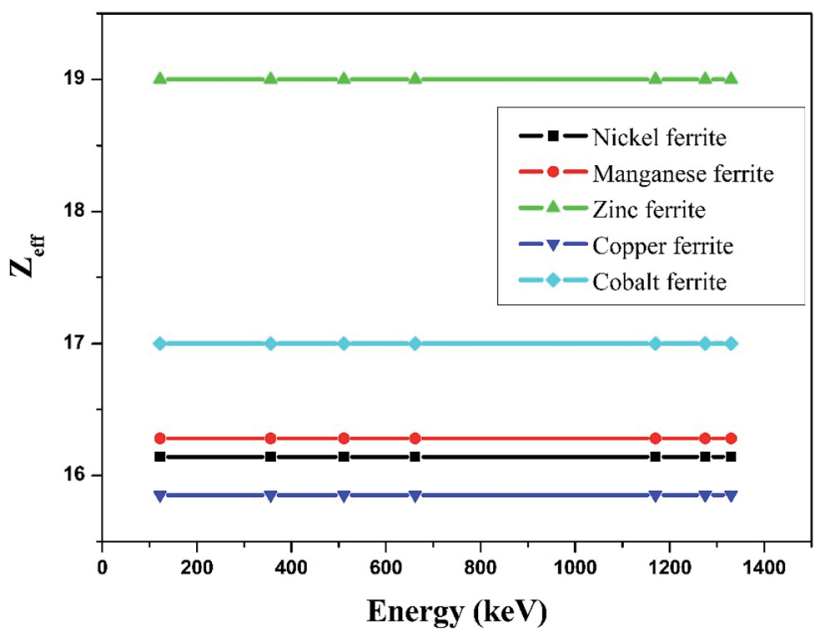

Fig. 7 Experimental study of effective atomic number $\left(Z_{\text {eff }}\right)$ against incident photon energy.

of the photons $(\sim 1330 \mathrm{keV})$. Zinc ferrite has the highest $Z_{\text {eff }}$ value compared with the other synthesized nano-ferrites, and manganese ferrite exhibits the lowest $Z_{\text {eff }}$ value. Fig. 8 demonstrates the dependence of effective electron density $\left(N_{\text {eff }}\right)$ on incident photon energy. $N_{\text {eff }}$ shows the same behavior as $Z_{\text {eff }}$ in the incident photon energy range of $122-1330 \mathrm{keV}$.

The half value layer (HVL), mean free path (mfp) and tenth value layer (TVL) are also vital parameters in predicting the gamma radiation shielding characteristics of the materials. In the present study, we have estimated the half value layer value utilizing relation (8).

Table 5 shows the half value layer parameter evaluated using Monte-Carlo simulation, XCOM and experimental data for the synthesized nano-ferrites. Fig. 9 shows the plots of HVL against incident photon energy for all the nano-ferrites. As the incident photon energy increases the half value layer value improves significantly. The zinc ferrite sample exhibits superior HVL values as compared to the other fabricated nano-ferrites. The

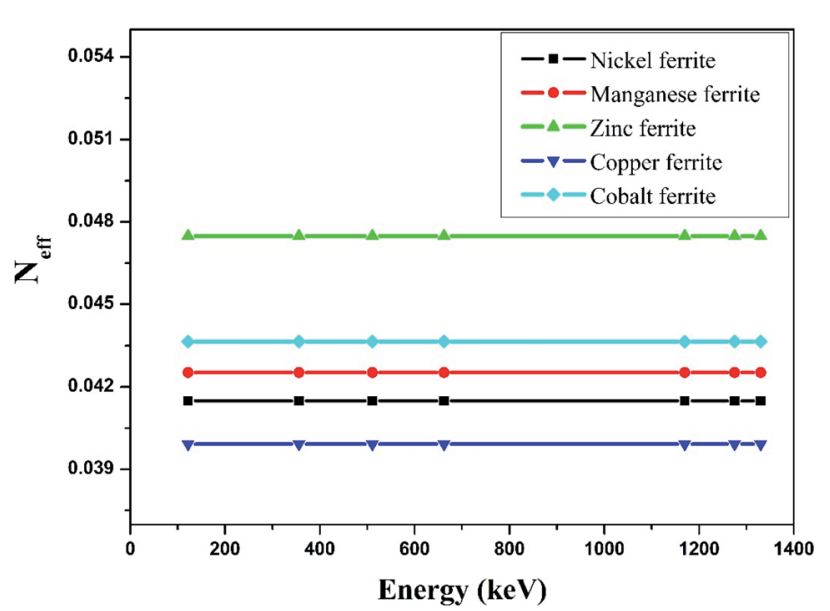

Fig. 8 Experimental study of effective electron density $\left(N_{\text {eff }}\right)$ against incident photon energy. 
Table 5 Half value layer for the synthesized spinel nano-ferrites

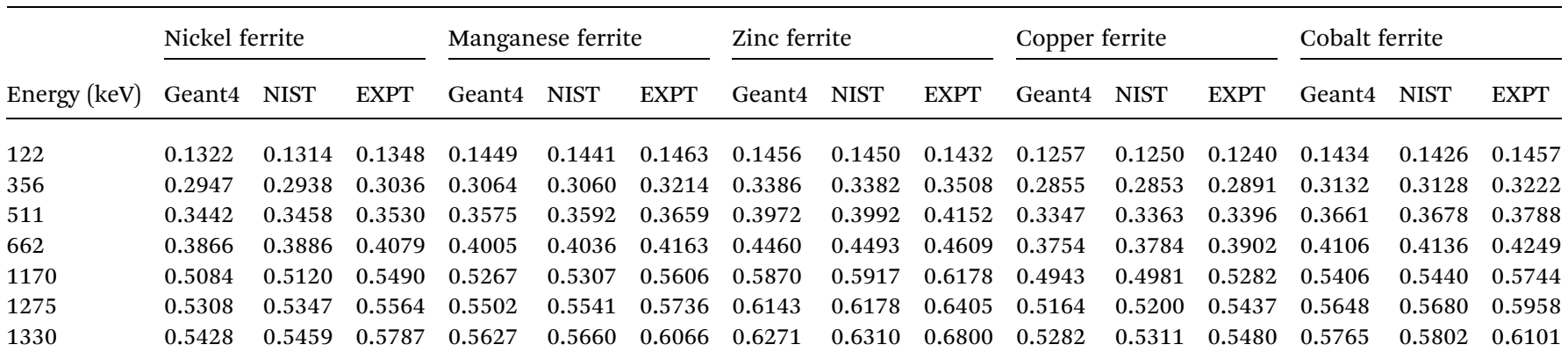

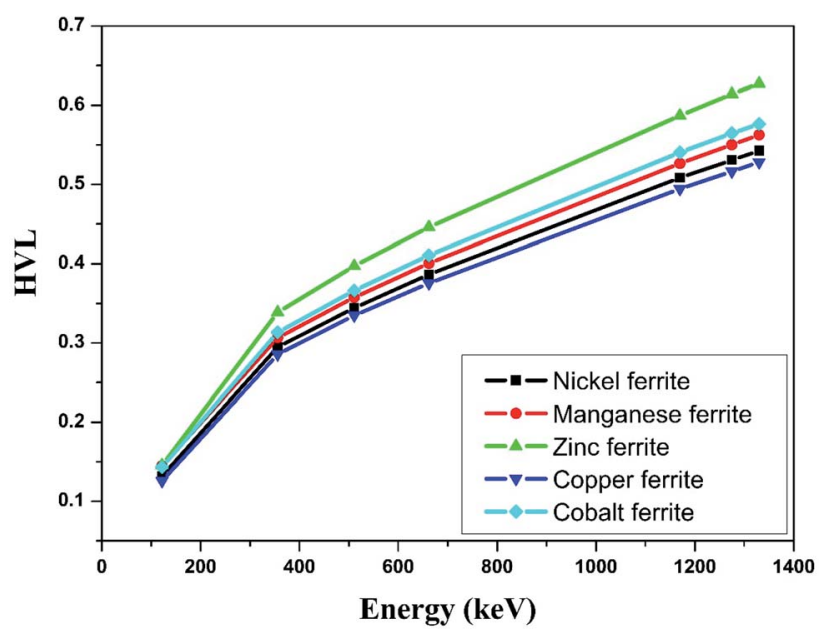

Fig. 9 Experimental study of half value layer against incident photon energy.

high HVL value of the synthesized nano-ferrites reflects the gamma ray shielding characteristics.

The equivalence atomic number $\left(Z_{\text {eq }}\right)$ of nano-ferrites at different photon energies was estimated using eqn (9) and is graphically presented in Fig. 10 . The $Z_{\text {eq }}$ value strongly relies on

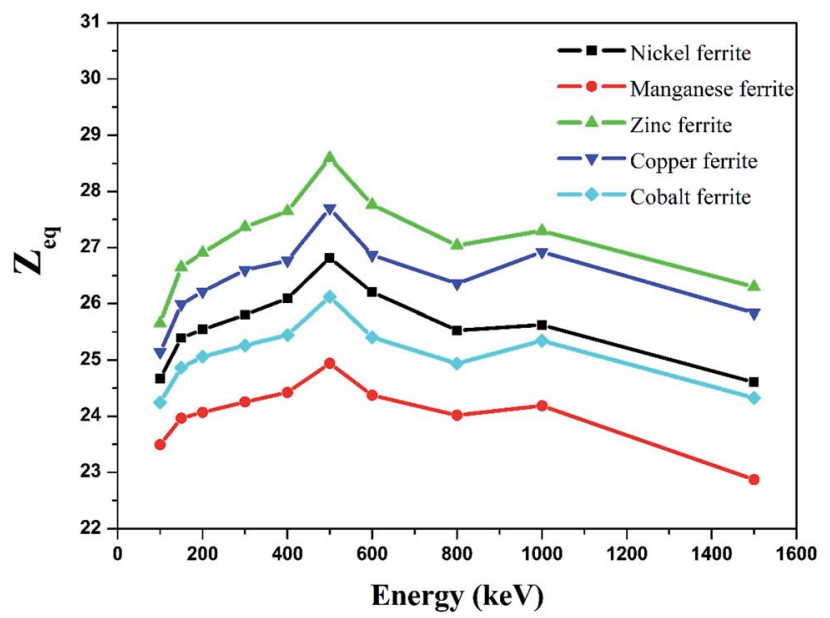

Fig. 10 Experimental study of equivalence atomic number $\left(Z_{\text {eq }}\right)$ against incident photon energy. the incident photon energy. The higher values of $Z_{\text {eq }}$ at the middle energy range are attributed to the Compton phenomenon, after that the lower or decreasing values of $Z_{\text {eq }}$ are attributed to the photoelectric process. The $Z_{\mathrm{eq}}$ is highest for zinc ferrite and lowest for manganese ferrite.

The variation in the energy absorption buildup factor (EABF) at an incident photon energy of $100-1500 \mathrm{keV}$ up to the penetration depth $1 \mathrm{mfp}, 10 \mathrm{mfp}, 20 \mathrm{mfp}, 40 \mathrm{mfp}$ was estimated using the G-P fitting method. The geometric progression parameter $a, b, c, d$ and $X_{\mathrm{k}}$ of spinel ferrites were determined by using ANSI/ANS-6.4.3-1991 data sheet and the interpolation formula developed by Harima. ${ }^{\mathbf{4 6 4 7}}$ With the help of Harima's interpolation formulae and ANSI 6.4.3 we also measured the EABF for all the nano-ferrites. Here, $b$ is the buildup factor of nano-ferrites and $k$ is a multiplication factor for dose at different penetration depths (when $k \neq 1$ ). Fig. 11(a-d) shows the variation in the EABF at different penetration depths viz. 1 $\mathrm{mfp}, 10 \mathrm{mfp}, 20 \mathrm{mfp}$ and $40 \mathrm{mfp}$ for all the produced spinel ferrite samples. All the spinel ferrites show unique behavior at different energy ranges. The curves of Fig. 11(a-d) reflect that, initially at the low energy region, the EABF increases with increasing photon energy, which is attributed to the dominance of the photoelectric effect, as the photoelectric process is directly proportional to the square of the atomic number and inversely to the cube of its energy. ${ }^{48}$

The EABF enhances significantly in the intermediate region of frequency which can be attributed to Compton process mainly because of multiple scattering of photons. Also, it is observed that the buildup factor value abruptly upsurges in the intermediate region for all penetration depths because of the generation of secondary gamma photons, which demonstrates multiple scattering. ${ }^{49}$ In the third region, as photon energy increases the EABF factor exhibits a simultaneous decreasing and increasing trend, $\sim 1000 \mathrm{keV}$, which can be mainly accredited to the pair production phenomenon.

Fig. 11(a-d) clearly depicts that the energy absorption buildup factor increases with increase in penetration depth from $1 \mathrm{mfp}$ to $40 \mathrm{mfp}$. Zinc ferrite exhibits the highest EABF value and manganese ferrite has the lowest EABF value at the penetration depth of $1 \mathrm{mfp}$ to $40 \mathrm{mfp}$ with photon energy of 100-1500 keV. In earlier reports, B. Singh et al. have examined the EABF value of low $Z_{\text {eq }}$ shielding materials and have observed that the materials with low $Z_{\text {eq }}$ exhibit a high EABF value. ${ }^{50}$ 


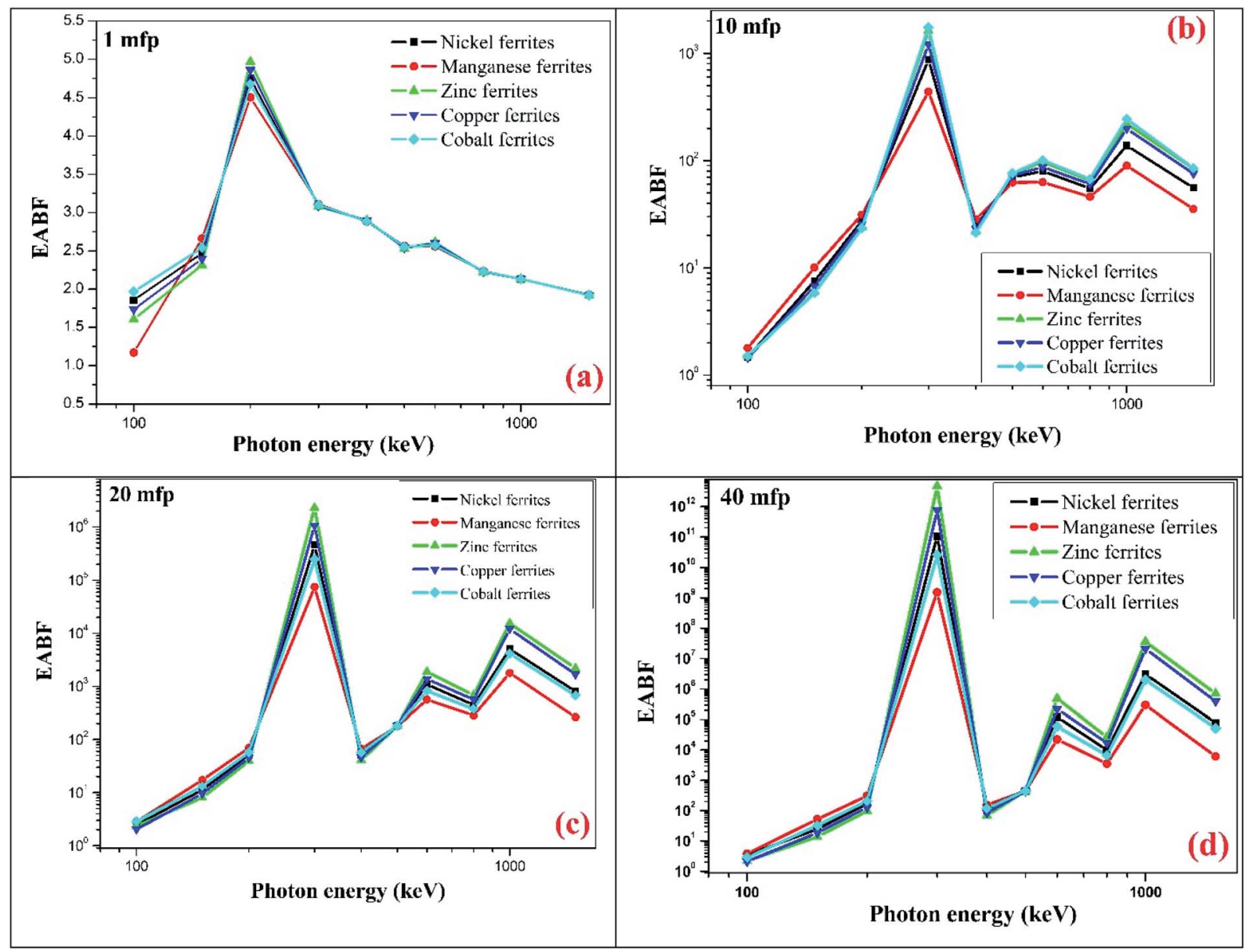

Fig. 11 Theoretical EABF values against incident photon energy up to penetration depth (a) $1 \mathrm{mfp}$ (b) $10 \mathrm{mfp}$ (c) $20 \mathrm{mfp}$ and (d) $40 \mathrm{mfp}$ of the synthesized ferrites at photon energy of 100 to $1500 \mathrm{keV}$.

However, our result shows a greater value of the EABF with higher value of $Z_{\text {eq. }}$. Zinc nano-ferrite exhibits a higher EABF compared with the other spinel ferrite materials.

The observed values of $Z_{\text {eq }}$ and EABF reflect that the EABF is dependent on the chemical composition of the materials. For better elucidation of the energy absorption buildup factor with different penetration depths of $1 \mathrm{mfp}, 5 \mathrm{mfp}, 10 \mathrm{mfp}, 20 \mathrm{mfp}, 30$ $\mathrm{mfp}$ and $40 \mathrm{mfp}$ at various photon energies, the EABF as a function of incident photon energy at $122 \mathrm{keV}, 356 \mathrm{keV}, 662$ $\mathrm{keV}$, and $1530 \mathrm{keV}$ is portrayed in Fig. 12(a-d). All the ferrite materials show the same trend, the EABF increases with increasing photon energy. The energy absorption buildup factor is the lowest at $122 \mathrm{keV}$ energy and highest at $356 \mathrm{keV}$.

Therefore, it can be concluded that nano-spinel ferrite materials are promising highly efficient shielding materials that can be used to reduce radiation dose. The EABF is an important parameter for the measurement of the radiation shielding properties of materials. The obtained EABF values of the present nano-ferrite samples were compared with those of the existing materials utilized for gamma radiation shielding, such as oxide glasses, bismuth borate glasses and marble (for typical 10 MFP). The current samples under investigation show nearly the same order of EABF values at lower photon energy. However, at higher incident photon energy the spinel ferrite nanocrystals show superior values compared with oxide glasses, bismuth borate glasses and marble materials. ${ }^{51-53}$

Also, zinc ferrite displays higher shieling parameter (such as linear attenuation coefficient, effective atomic number, and EABF) values as compared to the other spinel ferrites. This can be understood on the basis of the crystallite size effect; zinc ferrite possesses a lower crystallite size $(\sim 32.31 \mathrm{~nm})$ compared to nickel ferrite $(\sim 36.25 \mathrm{~nm})$, manganese ferrite $(\sim 33.87)$ copper ferrite $(\sim 39.75 \mathrm{~nm})$ and cobalt ferrite $(\sim 52.45 \mathrm{~nm})$. The obtained spinel ferrites in nano-dimensions are efficient in attenuating radiation since nanomaterials are more uniform and have less agglomeration in the matrix. Also, it is due to the fact that the cross section of photon interaction with material depends on the surface to volume ratio of nanoparticles. Hence, these materials can enhance the shielding ability of the electronic device.

This study reflects the high values of the energy absorption buildup factor for the fabricated spinel ferrite nanomaterials, which can be used for gamma ray shielding purposes, and the performance of electronic components based on spinel ferrite materials in gamma radiation atmosphere. 


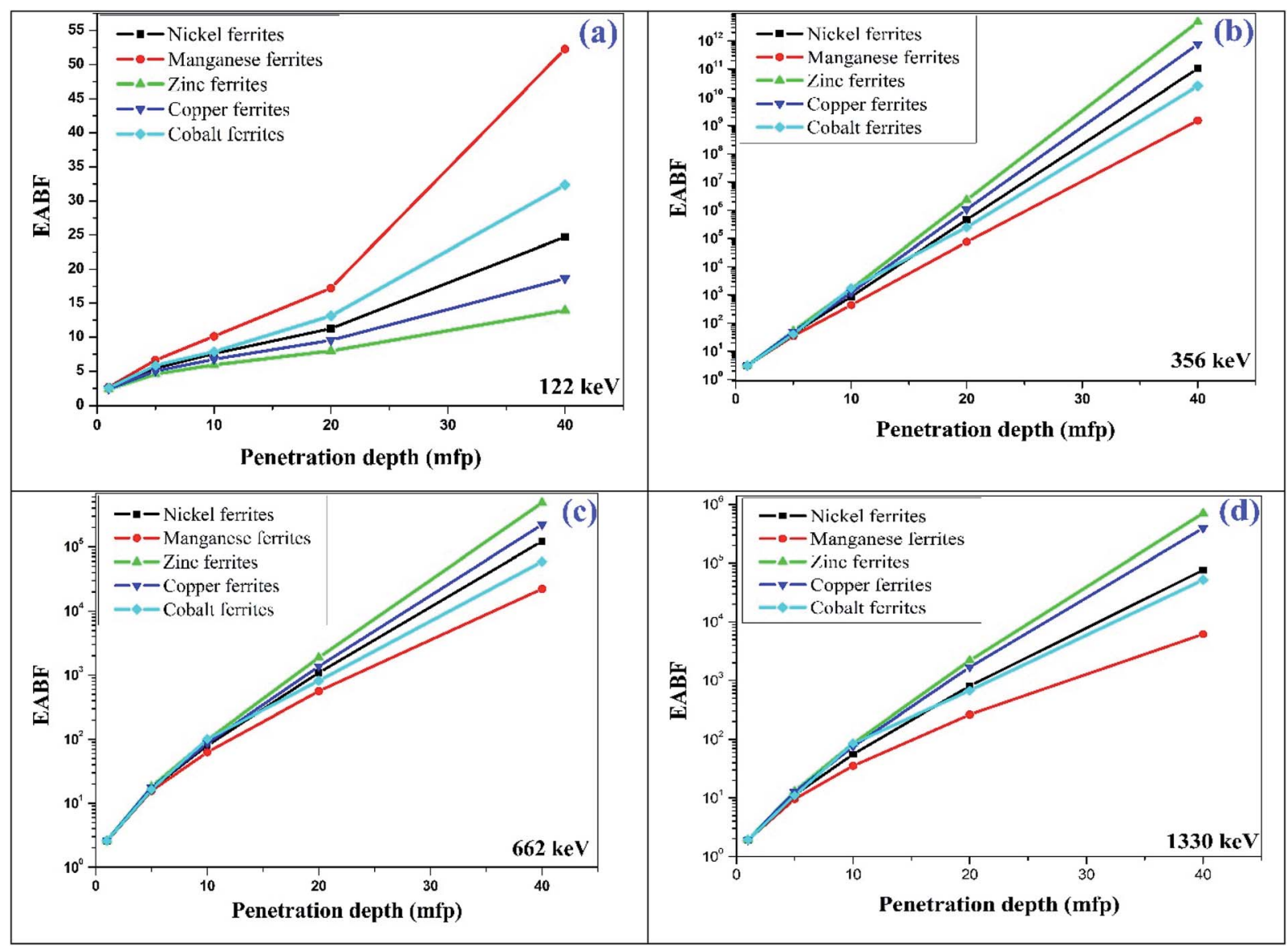

Fig. 12 Typical plot of penetration depth against EABF at (a) $122 \mathrm{keV}$, (b) $356 \mathrm{keV}$, (c) $662 \mathrm{keV}$ and (d) 1330 keV photon energy for the synthesized spinel ferrites.

\section{Conclusions}

In summary, spinel ferrite nanocrystals with high purity were effectively produced by the sol-gel auto-ignition route. Singlephase spinel ferrite nanocrystals of the cubic spinel $(F d \overline{3} m)$ phase were confirmed through X-ray analysis. The TEM outcomes showed the finely distributed uniform crystals in nano-dimensions. The mass attenuation coefficient of the ferrites was estimated at distinct incident photon energy in the range of $122-1330 \mathrm{keV}$. The mass attenuation coefficient, total atomic cross section, total electronic cross section and half value layer determined using Monto-Carlo simulation using Geant4, XCOM (NIST) and experimental procedure were in good agreement. The energy absorption buildup factor was measured at incident photon energy of $100-1500 \mathrm{keV}$ at penetration depths from 1 to $40 \mathrm{mfp}$ via the geometric progression (G-P) fitting method. The EABF values were mainly dependent on the chemical composition and $Z_{\text {eq }}$ of the material. Zinc ferrite displayed a higher EABF value and manganese ferrite had the lowest EABF value. Monte-Carlo simulation via Geant4 was found to be the most effective tool for theoretical parameter determination. Zinc ferrite displayed higher shieling parameters as compared to the other spinel ferrites. This can be understood on the basis of the crystallite size effect; zinc ferrite possesses a lower crystallite size $(\sim 32.31 \mathrm{~nm})$ compared to the other produced ferrites. This can be attributed to the homogenous distribution of nano-crystallites with high electron density, which results in higher interaction probability between incident photons and nanocrystals. This experimental and theoretical investigation suggests a new approach towards spinel ferrite nanomaterial at different penetration depths and different energy atmospheres. This gives vital information about the physico-chemical characteristics of spinel ferrites and their shielding properties upon interaction with gamma radiation, which can be applicable in the biomedical field, radiation protection for shielding purposes and performance of electronic appliances in radiation atmosphere.

\section{Conflicts of interest}

There are no conflicts to declare.

\section{Acknowledgements}

The authors are very much indebted to Department of Physics, IIT Mumbai for XRD facility, Sophisticated Analytical Instrument Facility (SAIF), IIT Bombay, Mumbai for TEM characterization, and Department of Physics, Dr Babasaheb Ambedkar Marathwada University for radiation measurements. 


\section{References}

1 B. Bhushan, Springer handbook of nanotechnology, Springer, 2017.

2 J. Lee, A. Yadav, M. Antia, V. Zaffino, E. Flitsiyan, L. Chernyak, J. Salzman, B. Meyler, S. Ahn and F. Ren, Low dose ${ }^{60} \mathrm{Co}$ gamma-irradiation effects on electronic carrier transport and DC characteristics of AlGaN/GaN highelectron-mobility transistors, Radiat. Eff. Defects Solids, 2017, 172, 250-256.

3 V. Vinayak, P. P. Khirade, S. D. Birajdar, R. Alange and K. Jadhav, Electrical and dielectrical properties of lowtemperature-synthesized nanocrystalline $\mathrm{Mg}$ 2+-substituted cobalt spinel ferrite, J. Supercond. Novel Magn., 2015, 28, 3351-3356.

4 M. Han and L. Deng, Doping effect of multiwall carbon nanotubes on the microwave electromagnetic properties of NiCoZn spinel ferrites, Appl. Phys. Lett., 2007, 90, 011108.

5 A. R. Chavan, S. B. Somvanshi, P. P. Khirade and K. Jadhav, Influence of trivalent $\mathrm{Cr}$ ion substitution on the physicochemical, optical, electrical, and dielectric properties of sprayed $\mathrm{NiFe}_{2} \mathrm{O}_{4}$ spinel-magnetic thin films, RSC Adv., 2020, 10, 25143-25154.

6 M. Pardavi-Horvath, Microwave applications of soft ferrites, J. Magn. Magn. Mater., 2000, 215, 171-183.

7 R. Valenzuela, Novel applications of ferrites, Phys. Res. Int., 2012, 2012.

8 J.-J. Lee, Y.-K. Hong, S. Bae, J.-H. Park, J. Jalli, G. S. Abo, R. Syslo, B.-C. Choi and G. W. Donohoe, High-quality factor Ni-Zn ferrite planar inductor, IEEE Trans. Magn., 2010, 46, 2417-2420.

9 S. B. Somvanshi, S. R. Patade, D. D. Andhare, S. A. Jadhav, M. V. Khedkar, P. B. Kharat, P. P. Khirade and K. Jadhav, Hyperthermic evaluation of oleic acid coated nano-spinel magnesium ferrite: enhancement via hydrophobic-tohydrophilic surface transformation, J. Alloys Compd., 2020, 835, 155422.

10 P. P. Khirade, Structural, microstructural and magnetic properties of sol-gel-synthesized novel $\mathrm{BaZrO}_{3}-\mathrm{CoFe}_{2} \mathrm{O}_{4}$ nanocomposite, J. Nanostruct. Chem., 2019, 9, 163-173.

11 G. J. Hine and G. L. Brownell, Radiation dosimetry, Elsevier, 2013.

12 K. Siegbahn, Alpha-, beta-and gamma-ray spectroscopy, Elsevier, 2012.

13 E. B. Podgoršak, Radiation physics for medical physicists, Springer, 2006.

14 H. Lischka, H. Henschel, O. Kohn, W. Lennartz and H. Schmidt, Radiation effects in light emitting diodes, laser diodes, photodiodes, and optocouplers, in RADECS 93. Second European Conference on Radiation and its Effects on Components and Systems (Cat. No. 93TH0616-3), IEEE, 1993, pp. 226-231.

15 E. H. Lee, A. Indluru, D. R. Allee, L. T. Clark, K. E. Holbert and T. L. Alford, Effects of gamma irradiation and electrical stress on a-Si: $\mathrm{H}$ thin-film transistors for flexible electronics and displays, J. Disp. Technol., 2011, 7, 325-329.
16 I. Fetahović, M. Pejović and M. Vujisić, Radiation damage in electronic memory devices, Int. J. Photoenergy, 2013, 2013.

17 H. Nikjoo, S. Uehara and D. Emfietzoglou, Interaction of radiation with matter, CRC press, 2012.

18 X. Li, C. Liu and J. Yang, Synergistic effect of ionization and displacement damage in NPN transistors caused by protons with various energies, IEEE Trans. Nucl. Sci., 2015, 62, 13751382.

19 (a) M. Rand and P. Schmidt, Radiation resistant coatings for semiconductor devices, US Pat. 3,765,935, Washington, DC, 1973; (b) S. J. Zinkle and L. L. Snead, Designing radiation resistance in materials for fusion energy, Annu. Rev. Mater. Res., 2014, 44, 241-267.

20 E. Little, Development of radiation resistant materials for advanced nuclear power plant, Mater. Sci. Technol., 2006, 22, 491-518.

21 S. S. Obaid, M. Sayyed, D. Gaikwad and P. P. Pawar, Attenuation coefficients and exposure buildup factor of some rocks for gamma ray shielding applications, Radiat. Phys. Chem., 2018, 148, 86-94.

22 R. M. Lokhande, C. V. More, B. S. Surung and P. P. Pawar, Determination of attenuation parameters and energy absorption build-up factor of amine group materials, Radiat. Phys. Chem., 2017, 141, 292-299.

23 A. H. Taqi and H. J. Khalil, An investigation on gamma attenuation of soil and oil-soil samples, J. Radiat. Res. Appl. Sci., 2017, 10, 252-261.

24 R. Kadam, S. Alone, G. Bichile and K. Jadhav, Measurement of atomic number and mass attenuation coefficient in magnesium ferrite, Pramana, 2007, 68, 869-874.

25 D. Creagh and J. Hubbell, Problems associated with the measurement of X-ray attenuation coefficients. I. Silicon. Report of the International Union of Crystallography X-ray Attenuation Project, Acta Crystallogr., Sect. A: Found. Crystallogr., 1987, 43, 102-112.

26 S. Raut, V. Awasarmol, S. Shaikh, B. Ghule, S. Ekar, R. Mane and P. Pawar, Study of gamma ray energy absorption and exposure buildup factors for ferrites by geometric progression fitting method, Radiat. Eff. Defects Solids, 2018, 173, 329-338.

27 S. Agostinelli, J. Allison, K. a. Amako, J. Apostolakis, H. Araujo, P. Arce, M. Asai, D. Axen, S. Banerjee and G. Barrand, GEANT4-a simulation toolkit, Nucl. Instrum. Methods Phys. Res., Sect. A, 2003, 506, 250-303.

28 T. Böhlen, F. Cerutti, M. Chin, A. Fassò, A. Ferrari, P. G. Ortega, A. Mairani, P. R. Sala, G. Smirnov and V. Vlachoudis, The FLUKA code: developments and challenges for high energy and medical applications, $\mathrm{Nucl}$. Data Sheets, 2014, 120, 211-214.

29 S. A. Pozzi, E. Padovani and M. Marseguerra, MCNP-PoliMi: a Monte-Carlo code for correlation measurements, Nucl. Instrum. Methods Phys. Res., Sect. A, 2003, 513, 550-558.

30 J. Allison, K. Amako, J. Apostolakis, P. Arce, M. Asai, T. Aso, E. Bagli, A. Bagulya, S. Banerjee and G. Barrand, Recent developments in Geant4, Nucl. Instrum. Methods Phys. Res., Sect. A, 2016, 835, 186-225. 
31 D. K. Gaikwad, P. P. Pawar and T. P. Selvam, Mass attenuation coefficients and effective atomic numbers of biological compounds for gamma ray interactions, Radiat. Phys. Chem., 2017, 138, 75-80.

32 P. Limkitjaroenporn, J. Kaewkhao, P. Limsuwan and W. Chewpraditkul, Physical, optical, structural and gamma-ray shielding properties of lead sodium borate glasses, J. Phys. Chem. Solids, 2011, 72, 245-251.

33 K. Singh, H. Singh, V. Sharma, R. Nathuram, A. Khanna, R. Kumar, S. S. Bhatti and H. S. Sahota, Gamma-ray attenuation coefficients in bismuth borate glasses, $\mathrm{Nucl}$. Instrum. Methods Phys. Res., Sect. B, 2002, 194, 1-6.

34 N. Singh, K. J. Singh, K. Singh and H. Singh, Gamma-ray attenuation studies of $\mathrm{PbO}-\mathrm{BaO}-\mathrm{B}_{2} \mathrm{O}_{3}$ glass system, Radiat. Meas., 2006, 41, 84-88.

35 M. Sayyed, M. AlZaatreh, M. Dong, M. Zaid, K. Matori and H. Tekin, A comprehensive study of the energy absorption and exposure buildup factors of different bricks for gamma-rays shielding, Results Phys., 2017, 7, 2528-2533.

36 Y. Harima, Y. Sakamoto, S. Tanaka and M. Kawai, Validity of the geometric-progression formula in approximating gamma-ray buildup factors, Nucl. Sci. Eng., 1986, 94, 24-35.

37 H. Saleh, J. Sharaf, S. Alkhateeb and M. Hamideen, Studies on equivalent atomic number and photon buildup factors for some tissues and phantom materials, Radiat. Phys. Chem., 2019, 165, 108388.

$38 \mathrm{H}$. Manjunatha and B. Rudraswamy, Computation of exposure build-up factors in teeth, Radiat. Phys. Chem., 2011, 80, 14-21.

39 D. Sardari and M. Kurudirek, A semi-empirical approach to the geometric progression (GP) fitting approximation in estimating photon buildup factor in soft tissue, water, and dosimetric materials, Int. J. Phys. Sci., 2012, 7, 5852-5860.

40 A. Raut, P. Khirade, A. Humbe, S. Jadhav and D. Shengule, Structural, electrical, dielectric and magnetic properties of $\mathrm{Al}^{3+}$ substituted Ni-Zn ferrite, J. Supercond. Novel Magn., 2016, 29, 1331-1337.

41 K. Mahmoud, O. Tashlykov, A. El Wakil and I. El Aassy, Aggregates grain size and press rate dependence of the shielding parameters for some concretes, Prog. Nucl. Energy, 2020, 118, 103092.
42 M. Medhat, N. Demir, U. Akar Tarim and O. Gurler, Calculation of gamma-ray mass attenuation coefficients of some Egyptian soil samples using Monte Carlo methods, Radiat. Eff. Defects Solids, 2014, 169, 706-714.

43 A. M. El-Khatib, M. I. Abbas, M. Abd Elzaher, M. S. Badawi, M. T. Alabsy, G. A. Alharshan and D. A. Aloraini, Gamma attenuation coefficients of nano cadmium oxide/high density polyethylene composites, Sci. Rep., 2019, 9, 1-11.

44 R. Li, Y. Gu, Y. Wang, Z. Yang, M. Li and Z. Zhang, Effect of particle size on gamma radiation shielding property of gadolinium oxide dispersed epoxy resin matrix composite, Mater. Res. Express, 2017, 4, 035035.

45 S. S. Obaid, D. K. Gaikwad and P. P. Pawar, Determination of gamma ray shielding parameters of rocks and concrete, Radiat. Phys. Chem., 2018, 144, 356-360.

46 J. Ryman, F. Alpan, L. Durani, K. Eckerman, R. Faw, L. Ruggieri and C. Sanders, Revision of ANSI/ANS-6.4. 3, Transactions, 2008, 99, 613-614.

47 Y. Yoshida, Development of fitting methods using geometric progression formulae of gamma-ray buildup factors, $\mathrm{J}$. Nucl. Sci. Technol., 2006, 43, 1446-1457.

48 S. Schön, Nuclear/Radioactive Properties, in Handbook of Petroleum Exploration and Production, Elsevier, 2011, pp. 107-148.

49 M. Halimah, A. Azuraida, M. Ishak and L. Hasnimulyati, Influence of bismuth oxide on gamma radiation shielding properties of boro-tellurite glass, J. Non-Cryst. Solids, 2019, 512, 140-147.

50 T. Singh, N. Kumar and P. S. Singh, Chemical composition dependence of exposure buildup factors for some polymers, Ann. Nucl. Energy, 2009, 36, 114-120.

51 S. Gupta and G. S. Sidhu, Energy absorption buildup factor for some oxide glasses: penetration depth, photon energy and effective atomic number dependence, Int. J. Sci. Res., 2012, 2, 1-7.

52 M. S. Al-Buriahi and B. T. Tonguc, Study on gamma-ray buildup factors of bismuth borate glasses, Appl. Phys. A, 2019, 125, 1-7.

53 M. Büyükyıldız, A. Kılıç and D. Yılmaz, White and some colored marbles as alternative radiation shielding materials for applications, Radiat. Eff. Defects Solids, 2020, 175, 657-671. 